\title{
TOWARD A PROOF OF LONG RANGE ORDER IN 4-D SU(N) LATTICE GAUGE THEORY
}

\author{
Michael Grady \\ Department of Physics \\ State University of New York at Fredonia \\ Fredonia NY 14063 USA \\ ph:(716)673-4624, fax:(716)673-3347, email: grady@fredonia.edu
}

\begin{abstract}
An extended version of 4-d SU(2) lattice gauge theory is considered in which different inverse coupling parameters are used, $\beta_{H}=4 / g_{H}^{2}$ for plaquettes which are purely spacelike, and $\beta_{V}$ for those which involve the Euclidean timelike direction. It is shown that when $\beta_{H}=\infty$ the partition function becomes, in the Coulomb Gauge, exactly that of a set of non-interacting 3-d $\mathrm{O}(4)$ classical Heisenberg models. Long range order at low temperatures (weak coupling) has been rigorously proven for this model. It is shown that the correlation function demonstrating spontaneous magnetization in the ferromagnetic phase is a continuous function of $g_{H}$ at $g_{H}=0$ and therefore that the spontaneously broken phase enters the $\left(\beta_{H}, \beta_{V}\right)$ phase plane (no step discontinuity at the edge). Once the phase transition line has entered, it can only exit at another identified edge, which requires the $\mathrm{SU}(2)$ gauge theory within also to have a phase transition at finite $\beta$. A phase exhibiting spontaneous breaking of the remnant symmetry left after Coulomb gauge fixing, the relevant symmetry here, is non-confining. Easy extension to the $\mathrm{SU}(\mathrm{N})$ case implies that the continuum limit of zero-temperature 4-d SU(N) lattice gauge theories is not confining, in other words gluons by themselves do not produce a confinement.
\end{abstract}

Keywords: lattice gauge theory, phase transition, confinement, long range order

PACS Nos.: 11.15.Ha, 11.30.Qc, 64.60.De, 5.50.+q

\section{Introduction}

For many years it has been believed that $\mathrm{SU}(\mathrm{N})$ lattice gauge theories exist in a single phase. Since confinement is a property of all compact lattice gauge theories at strong coupling, this assumption has the consequence that these theories also have a confining force (linear potential) in the weak coupling continuum limit. This fits rather nicely with phenomenological evidence that the strong interactions confine quarks. The numerical evidence for lack of a phase transition in $\mathrm{SU}(2)$ comes mostly from the apparently smooth behavior of the specific heat as a function of the coupling parameter, $\beta$. However, if the critical exponent $\nu$ is large enough to produce a large negative specific heat exponent $\alpha(\alpha=2-d \nu)$, then the first infinite singularity may be in a high derivative, and not easily visible numerically. This is, for instance, often the case for 3-d spin glasses which are close to their lower critical dimension. The argument in favor of the existence a phase transition rests on the similarity of lattice gauge theories to ferromagnetic spin models, all of which in three or more dimensions, at least for short range interactions, have two phases, 
with the weak coupling phase exhibiting spontaneous symmetry breaking and long range order. Abelian and non-abelian spin models differ in detail, but all have phase transitions. A major difference between gauge models and spin models are that the former have local symmetries and the latter global. This difference can sometimes be erased through gauge fixing, however. For instance the 2-d lattice gauge theory is exactly equal to a set of non-interacting 1-d classical spin chains in the axial gauge, neglecting possible boundary effects. Setting all of the links in one direction equal to unity using the gauge freedom causes the 4-link plaquette action to collapse to a two-link dot product between the remaining gauge links which can be reinterpreted as spins! 1 Another exact mapping is between the 3 -d Ising gauge theory in axial gauge with the 3 -d Ising model ${ }^{2}$ For 4 -d theories, the Coulomb gauge appears to be the most "spin-like." Here the gauge freedom is used to maximize the traces of all of the links lying along three of the four lattice directions. In this gauge the fourth direction lying links become observables and act much like spins, in that they transform under a remnant 3-d global symmetry on each spacelike hyperlayer that remains after Coulomb gauge fixing. This symmetry can break spontaneously if the average of fourth-direction links over a hyperlayer acquires a magnetization. It has been shown that if this symmetry does break spontaneously, then the corresponding ferromagnetic phase is non-confining, so the average of these 4-th direction links serves as a local order parameter for confinement 3445 According to the standard hypothesis, the theory remains in the paramagnetic phase for all couplings in the non-abelian case, and undergoes a magnetic phase transition only in the abelian case, such as U(1). However, below it will be shown that this inconsistent with the known long-range order for classical 3-d Heisenberg models, to which the 4-d lattice gauge theories are closely related and actually connected in a larger coupling space.

Numerical evidence for a ferromagnetic phase transition based on the behavior of the Coulomb gauge spin-like order parameter described above has been given for $\mathrm{SU}(2)$ with the Wilson action. Finite size scaling shows an infinite-lattice transition around $\beta=3.2$ with a correlation length critical exponent $\nu=1.7 \pm 0.2 .6$ Other simulations $\sqrt{6}$ which supplement the Wilson action with an infinite chemical potential for gauge-invariant $\mathrm{SO}(3)-\mathrm{Z} 2$ monopoles 7 , together with a positive plaquette constraint, appear to remain in the ferromagnetic phase for all couplings. This would seem to indicate that the normal confinement seen with the Wilson action is due to strong-coupling lattice artifacts, not unlike the U(1) case. In the following, the analytic case for the existence of this phase transition is explored. An analytic proof for the opposite hypothesis, namely the lack of a phase transition with confinement persisting to the continuum limit, was presented by Tomboulis some time agd 8 and updated more recently $\frac{9}{9}$ This proof is fairly complex and some possible flaws have been noted 10 When an hypothesis is difficult to prove, it is sometimes worthwhile to attempt to prove the opposite, which is the approach taken below. 


\section{Extended Coupling Plane}

The 4-d SU(2) theory can be extended into a larger coupling space by allowing the coupling for purely spacelike (horizontal) plaquettes to differ from that of plaquettes which include the Euclidean timelike direction (vertical). The action is

$$
\begin{aligned}
S= & \sum_{\vec{n}}\left(\beta_{H} \sum_{i=1}^{2} \sum_{j=i+1}^{3}\left(1-\frac{1}{2} \operatorname{tr}\left(U_{\vec{n}, i} U_{\vec{n}+\hat{\imath}, j} U_{\vec{n}+\hat{\jmath}, i}^{\dagger} U_{\vec{n}, j}^{\dagger}\right)\right)\right. \\
& \left.+\beta_{V} \sum_{i=1}^{3}\left(1-\frac{1}{2} \operatorname{tr}\left(U_{\vec{n}, i} U_{\vec{n}+\hat{\imath}, 4} U_{\vec{n}+\hat{4}, i}^{\dagger} U_{\vec{n}, 4}^{\dagger}\right)\right)\right)
\end{aligned}
$$

Where the $U_{\vec{n}, j}$ are $\mathrm{SU}(2)$ valued gauge links based at lattice site $\vec{n}$ in direction $j$. The normal Wilson action is simply the $\beta_{V}=\beta_{H}$ case, however because the renormalization group gives a relation between $\beta$ and the physical lattice spacing through the running coupling, the $\beta_{V} \neq \beta_{H}$ theory can also be considered an $\mathrm{SU}(2)$ LGT with unequal lattice spacings in the spacelike and Euclidean timelike directions. So at least in the phase connected to the continuum limit (neighborhood of $\left.\left(\beta_{H}, \beta_{V}\right) \rightarrow(\infty, \infty)\right)$ the entire phase plane excluding the boundaries can be considered an $\mathrm{SU}(2) \mathrm{LGT}$.

Consider now the $\beta_{H}=\infty$ theory. This can be seen to be equivalent to a set of non-interacting 3-d $\mathrm{O}(4)$ classical Heisenberg models as follows. At $\beta_{H}=\infty$ all of the horizontal plaquettes will be forced to their largest possible value of unity. One can then find a gauge in which all horizontal links are unity also, as follows. Set a maximal tree of links to unity on each spacelike hypersurface. This is a partial axial gauge in which the final trunk of the tree along the Euclidean timelike direction is not completed. For instance for an $L^{4}$ lattice, on a given spacelike hyperlayer all 3-direction links can be set to unity except when $z=0$. On the $z=0$ plane all 2-direction links are set to unity except for when $y=0$ and along the line $(y=0, z=0)$ all 1-direction links except for when $x=0$ can be set to unity by gauge transformations. Looking at the $z=0$ plane, there is a set of $x$ - $y$ plaquettes extending backward from $x=0$ (through the periodic boundary condition) which have three links set to unity. The plaquette being unity due to $\beta_{H}=\infty$ ensures that the fourth link in the plaquette is also unity. Now the same is true for the next row of plaquettes etc., forcing all links to unity except for the last row pointing in the positive direction from $\mathrm{x}=0$. These are now equal to the gauge invariant Polyakov loop for that direction, and all of these links are equal. This same procedure can be extended from the $(x=0, y=0)$ plane along the $z$ direction to show that the links out of the plane are also unity, except for one at the edge along each lattice direction which are equal to their neighboring links pointing in that direction. Finally, a Polyakov loop symmetry transformation (also a symmetry of the action) can be employed to bring these final set of links to unity. In this restricted sector, the Polyakov loop symmetry is the whole $\mathrm{SU}(2)$ group rather than just the center. One could also formulate the theory with open boundary conditions, in which case 
this final step would be unnecessary. Although the language of axial gauge was used above, one can see that actually the condition for Coulomb gauge has also been met, that the sum of traces of all horizontal links is maximized. Coulomb gauge leaves a layered remnant symmetry, one global SU(2) per hyperlayer, unfixed. Because Coulomb gauge was being sought is why the axial tree above was left uncompleted.

Since all of the horizontal plaquettes are unity, the vertical plaquettes simplify to

$$
\frac{1}{2} \operatorname{tr}\left(U_{\vec{n}, 4}^{\dagger} U_{\vec{n}+\hat{\jmath}, 4}\right)
$$

where $j$, of course, can be 1,2 or 3 . Writing each $U$ as

$$
U=s_{0}+i \sum_{k=1}^{3} s_{k} \tau_{k}
$$

where the $\tau_{k}$ are the Pauli matrices, one can associate an $\mathrm{O}(4)$ unit vector $\vec{s}=\left(s_{0}\right.$, $\left.s_{1}, s_{2}, s_{3}\right)$ with the $\mathrm{SU}(2)$ valued link. In terms of the $\vec{s}$ 's it is easily verified that the vertical plaquette above is simply the nearest neighbor $\mathrm{O}(4)$ dot product,

$$
\frac{1}{2} \operatorname{tr}\left(U_{\vec{n}, 4}^{\dagger} U_{\vec{n}+\hat{\jmath}, 4}\right)=\vec{s}_{\vec{n}} \cdot \vec{s}_{\vec{n}+\hat{\jmath}}
$$

The action on each hyperlayer is that of the 3 -d $\mathrm{O}(4)$ Heisenberg model with coupling parameter (inverse temperature) $\beta_{V}$. In a gauge theory there are never any direct interactions between links longitudinally, and the freezing of horizontal links prevents any indirect interactions among hyperlayers, so these all become independent Heisenberg models. Each of these has its own $\mathrm{SU}(2)$ global symmetry, the remnant symmetry from the Coulomb gauge. The reason behind this is that an $\mathrm{SU}(2)$ gauge transformation which is global on the hyperlayer transforms horizontal links in such a way as to leave the trace of each link unchanged. Thus the gauge condition, which is to maximize these traces, is not disturbed. Therefore, at the level of the partition function, the $\beta_{H}=\infty \mathrm{SU}(2)$ lattice gauge theory becomes a set of non-interacting 3-d O(4) classical Heisenberg Models. Durhus and Fröhlich 11 earlier pointed out a connection between $\mathrm{SU}(2)$ lattice gauge theory and the 3-d $\mathrm{O}(4)$ model, but did not consider the case of split vertical and horizontal couplings which allows for an exact mapping.

Long range order (LRO) in the classical Heisenberg model has been rigorously proven $[12$ This means that a ferromagnetic phase must exist at a finite weak coupling (large $\beta_{V}$ ). Because this is a symmetry broken phase, it must be separated from the strong-coupling paramagnetic phase by a phase transition, which has been convincingly found by Monte Carlo simulation at a coupling $\beta=0.9360(1) ! 13$ The LRO proof does not depend on the specific symmetry group. Indeed, the existence of phase transitions in all ferromagnetic spin models is well-established and noncontroversial from both analytic and numerical perspectives. The ferromagnetic order for the Heisenberg model is also quite robust to the addition other interactions. For instance it is still preserved at zero temperature even if up to $20 \%$ of the 
interactions are switched to anti-ferromagnetic (beyond this level it enters a spin glass phase $)^{14}$, and at higher temperatures with smaller but still substantial contaminations. Below, it will be argued that the ferromagnetic phase, and therefore also the phase transition, continues to exist for non-infinite $\beta_{H}$ as well.

In Fig. 1 the $\beta_{H}-\beta_{V}$ phase plane is shown, with the $3-\mathrm{d} \mathrm{O}(4)$ model existing on the top edge $\left(\beta_{H}=\infty\right)$. The normal 4-d SU(2) model exists on the $\beta_{V}=\beta_{H}$ line. However, as stated before, the $\beta_{V} \neq \beta_{H}$ cases can also be considered to be $\mathrm{SU}(2)$ lattice gauge theories, with a different lattice spacing in the fourth direction than the other three. Thus the entire interior, at least in the vicinity of the continuum limit in the upper right corner, is an $\mathrm{SU}(2)$ lattice gauge theory.

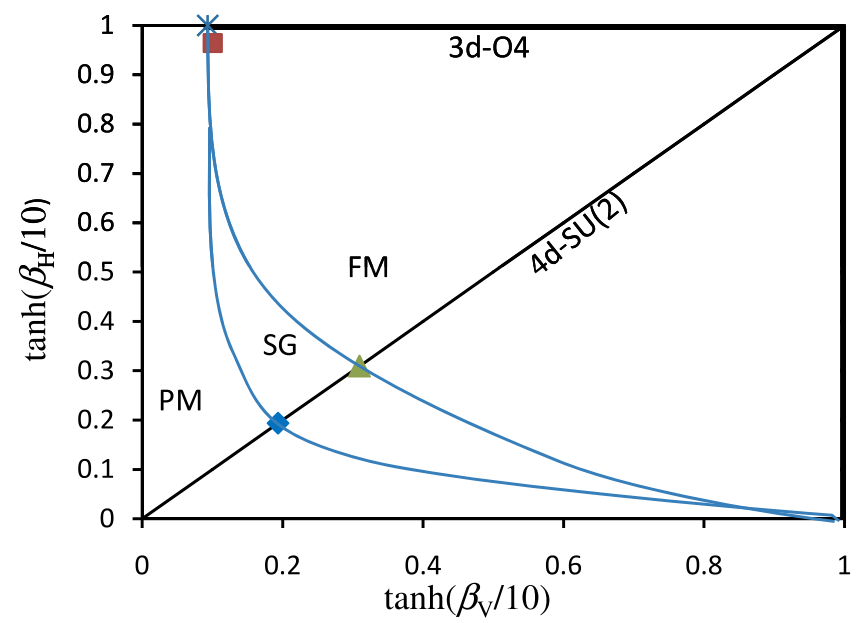

Fig. 1. Possible phase diagram for $\mathrm{SU}(2)$ lattice gauge theory with different horizontal and vertical couplings. Top horizontal axis is 3-d O (4) Heisenberg model and the star shows its known ferromagnetic phase transition 13 Square and triangle show locations of phase transitions at $\beta_{H}=20$ and $\beta_{H}=\beta_{V}$ found from Monte Carlo simulations using the Coulomb gauge magnetization $[6]$ Diamond is possible spin-glass to paramagnetic transition found previously using the real replica method!15 Bold regions of upper and right edges show portions of the border known to be ferromagnetic. Lines drawn are hypothetical phase boundaries guided by the Monte Carlo results.

The bottom edge, with $\beta_{H}=0$, has only the vertical plaquettes left. In an axial gauge with the fourth direction links set to unity, the system becomes a set of 1-d spin chains, which are paramagnetic except at zero temperature $\left(\beta_{V}=\infty\right)$ where there is a phase transition to the ferromagnetic ground state. The right edge of the phase diagram, $\beta_{V}=\infty$, is an odd phase where all of the vertical plaquettes are forced to unity. Staying with the axial gauge, one can see that the 3-d SU(2) theories on spacelike hypersurfaces but different Euclidean times are locked to each other, in other words they can only fluctuate in lockstep. This makes for a theory with a 4-d energy, but non-extensive 3 -d entropy, so it is basically stuck in the 
classical ground state. Both top and bottom ends of this line are ferromagnetic; there is no reason for the whole line not to be also. Thus ferromagnetism exists on the upper border for $\beta_{V}>0.936$ and along the entire right-side border. In the following, two arguments are given which show that the ferromagnetic phase persists as one enters the interior of the phase diagram. An important point is that the global SU(2) symmetries of the independent Heisenberg models persist for the $\beta_{H}<\infty$ case in the form of the Coulomb Gauge remnant symmetries which exist independently on each hyperlayer. For a symmetry breaking phase transition, once the phase-transition line has entered the phase plane it must continue to another edge. This is because the order parameter, due to the realized symmetry, is exactly zero in the paramagnetic region on the infinite lattice, and of course is nonzero in the ferromagnetic phase. An analytic function zero in a finite region is zero everywhere, so a line of non-analyticity must separate the two phases completely. The only place where the phase transition line could terminate is at the only other phase transition on the border, namely the lower right corner as shown. Because the remnant symmetry exists for both the $\beta_{H}=\infty$ and the $\beta_{H}$ finite cases, this situation is not analogous to the Ising model in an external field, where the field, no matter how small, removes the transition due to explicit symmetry breaking.

Fig. 1 also shows the ferromagnetic phase transitions found with Monte Carlo simulations using the Coulomb Gauge magnetization as the order parameter. One of these was for $\beta_{H}=20$ where the phase transition on the infinite lattice was determined to be $\beta_{V c}=1.01(2)$. This transition closely resembles that of the Heisenberg model in that it has similar critical exponents. Finite size scaling fits are good, which indicates lattices are large enough to suppress non-leading effects. Observation of such a transition in Monte Carlo simulations is a strong indication that the phase transition does enter the interior of the phase diagram. Below, it will be argued that that must be the case from an analytic perspective as well.

\section{Persistence of Phase Transition for $\boldsymbol{\beta}_{\boldsymbol{H}}<\infty$}

In order for the $\mathrm{SU}(2)$ lattice gauge theory in the interior of the phase diagram to avoid the phase transition as in the conventional hypothesis, the transition would need to be somehow prevented from entering the interior of the phase diagram at all. This means that the small terms that arise in the Hamiltonian when $\beta_{H}$ is backed off from $\infty$ would have to destroy the ferromagnetic order, no matter how small the coupling $g_{H}\left(\beta_{H}=4 / g_{H}^{2}\right)$. This seems odd considering the continued existence of the symmetry and the known robustness of ferromagnetism in the Heisenberg model. Say we start in the deep-ferromagnetic region of the Heisenberg model. For the order parameter to jump from a finite value at $g_{H}=0$ to a value of zero for any $g_{H}>0$ there would have to be a first-order phase transition at the edge of the phase diagram. However, this edge is already well characterized, since it is the ferromagnetic phase of the Heisenberg model itself. Known properties of this phase are inconsistent with it being the location of a first-order phase transition in the 
same order parameter due to the following. A symmetry-breaking first-order phase transition is of the type associated with a tricritical point, which is controlled by a sixth-order Landau effective potential as shown in Fig. 2 (solid line) at the point of phase transition. $\frac{16}{16}$ For $g_{H}>0$ the two side-minima would hypothetically lift up, leaving only the minimum at zero order parameter. On the phase transition point, both the phase with zero order parameter and the two instances of the brokensymmetry phase exist in equilibrium. Such phase mixing would be easily observable through an un-sharp order parameter and a latent heat (range of internal energies). However, such a state simply does not exist in the Heisenberg model. In the deep ferromagnetic region its Landau effective potential is widely believed to look like the dashed line in Fig. 2 with only two minima which is incompatible with the tricritical behavior.

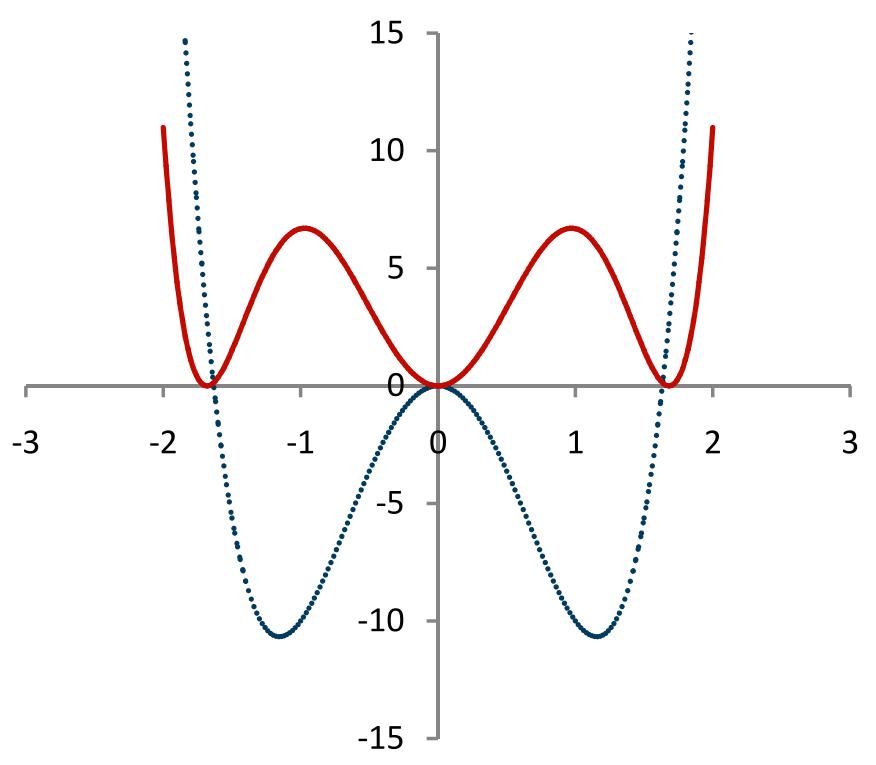

Fig. 2. Landau effective potentials at a symmetry breaking first-order transition (solid line) and in the deep-ferromagnetic region of a system with a higher-order transition (dotted line). Scales are arbitrary.

Therefore, the hypothesis that the ferromagnetic phase does not enter the interior of the phase diagram seems to require behavior of the Heisenberg model inconsistent with known behavior. If a first-order phase transition is present it cannot be a conventional one. However, the argument above is somewhat heuristic and non-rigorous in that it involves Landau effective potentials. A more rigorous proof of the impossibility of a first-order phase transition as one enters the phase plane from the deep-ferromagnetic region of the Heisenberg model can be constructed as follows. Consider the correlation function $C(\vec{q}, \vec{r})=<\frac{1}{2} \operatorname{tr}\left(U_{\vec{q}, 4} U_{\vec{r}, 4}^{\dagger}\right)>$. In the 
Michael Grady

ferromagnetic phase this shows spontaneous symmetry breaking through

$$
\lim _{|\vec{q}-\vec{r}| \rightarrow \infty} C(\vec{q}, \vec{r})=<|\vec{m}|>^{2}
$$

where

$$
\vec{m}=\frac{1}{V} \sum_{\vec{n}} \vec{s}_{\vec{n}}
$$

where the sum is over individual 3 -d hypersurfaces and $V$ is the 3 -volume. The expectation value above includes an average over different Euclidean times as well as gauge configurations. Although a finite lattice is used for defining quantities, the infinite lattice case is implicitly being considered here.

Now let us calculate the derivative of this correlation function with respect to $g_{H}$ at $g_{H}=0$. Note that the calculation is performed in the Heisenberg model itself (earlier shown to be the $g_{H}=0$ limit), but in order to determine what function to calculate the expectation value of, we must consider the $g_{H} \neq 0$ theory. To do this, rewrite the horizontal $U$ links in terms of gauge fields $A$, as in the usual calculation of the continuum limit:

$$
\begin{aligned}
S= & \sum_{\vec{n}}\left(\frac { 4 } { g _ { H } ^ { 2 } } \sum _ { i , j > i } \left(1-\frac{1}{2} \operatorname{tr}\left(\exp \left(i g_{H} \vec{A}_{\vec{n}, i} \cdot \vec{\tau}\right) \exp \left(i g_{H} \vec{A}_{\vec{n}+\hat{\imath}, j} \cdot \vec{\tau}\right)\right.\right.\right. \\
& \left.\left.\quad \exp \left(-i g_{H} \vec{A}_{\vec{n}+\hat{\jmath}, i} \cdot \vec{\tau}\right) \exp \left(-i g_{H} \vec{A}_{\vec{n}, j} \cdot \vec{\tau}\right)\right)\right) \\
& \left.+\beta_{V} \sum_{i<4}\left(1-\frac{1}{2} \operatorname{tr}\left(\exp \left(i g_{H} \vec{A}_{\vec{n}, i} \cdot \vec{\tau}\right) U_{\vec{n}+\hat{\imath}, 4} \exp \left(-i g_{H} \vec{A}_{\vec{n}+\hat{4}, i} \cdot \vec{\tau}\right) U_{\vec{n}, 4}^{\dagger}\right)\right)\right)
\end{aligned}
$$

where $i, j$ run from 1 to 3 . No approximation has been made. For finite $g_{H}$ this is not a practical decomposition, since the A integrations still need to obey the Harr measure for the $U$ 's, however it is useful in the limit $g_{H} \rightarrow 0$. Taking the limit $g_{H} \rightarrow 0$ gives

$$
S=\sum_{\vec{n}}\left(\sum_{i, j>i} F_{i j}^{2}+\beta_{V} \sum_{i}\left(1-\frac{1}{2} \operatorname{tr}\left(U_{\vec{n}+\hat{\imath}, 4} U_{\vec{n}, 4}^{\dagger}\right)\right)\right)
$$

where $F_{i j}$ is the abelian field strength tensor (the non-abelian part having a factor of $g_{H}$ ). This gives the multiple 3-d Heisenberg models as expected, but also a disconnected set of 3-d free field theories. The connected part of

$$
\left.\frac{\partial C(\vec{q}, \vec{r})}{\partial g_{H}}\right|_{g_{H}=0}
$$

is given by

$$
\frac{1}{4} \beta_{V} \sum_{\vec{n}, i<4}<\operatorname{tr}\left(U_{\vec{q}, 4} U_{\vec{r}, 4}^{\dagger}\right) \operatorname{tr}\left(\left(i \vec{A}_{\vec{n}, i} \cdot \vec{\tau}\right) U_{\vec{n}+\hat{\imath}, 4} U_{\vec{n}, 4}^{\dagger}\right)>
$$


and a similar term with the $\vec{A}$ in the other position. This is to be computed in the $g_{H}=0$ action of Eq. 8 which is even in the transformation $\vec{A} \rightarrow-\vec{A}$. As a consequence, any expectation value containing a single factor of $\vec{A}$ is zero. Disconnected parts similarly vanish or cancel. This almost trivial observation means that the first derivative of the order parameter with respect to $g_{V}$ at $g_{V}=0$ vanishes, and therefore exists. If the first derivative exists at a point, it follows from an elementary theorem of analysis that the function itself is continuous at that point $\frac{17}{17}$ However, if the order parameter is a continuous function of $g_{V}$ at $g_{V}=0$ then it cannot drop suddenly to zero here in a first-order phase transition, which would require a discontinuity. Therefore, the ferromagnetic phase must enter the phase diagram. In fact, since $g_{H}$ does not affect the phase transition at lowest order, the line would be expected to enter at a $90^{\circ}$ angle.

Since, as argued before, the entire interior of the phase diagram is an $\mathrm{SU}(2)$ LGT if unequal lattice spacings are considered, this means that there must be a ferromagnetic phase in $\mathrm{SU}(2)$ as well. The $\mathrm{SU}(2)$ LGT has long-range order. The order parameter is the Coulomb-gauge magnetization. As argued before, due to the symmetry breaking nature of the phase transition, the phase transition line must continue to another edge, the only possibility being the lower right corner of Fig. 1. In doing so it clearly must cross the $\beta_{V}=\beta_{H}$ line, which is the ordinary SU(2) LGT with equal lattice spacings. A phase transition in which the Coulomb Gauge remnant symmetry breaks spontaneously is known to be deconfining $\underline{3.415}$ Therefore the zero physical temperature (infinite lattice) 4-d SU(2) lattice gauge theory must have a deconfining phase transition, contrary to the usual assumption. To prove for $\mathrm{SU}(3)$ or $\mathrm{SU}(\mathrm{N})$ one only has to replace the $\mathrm{O}(4)$ Heisenberg model with the $\mathrm{SU}(\mathrm{N}) \times \mathrm{SU}(\mathrm{N})$ spin model. Since these all have ferromagnetic phases, the argument goes through in the same way for them.

This argument has similarities to another approach which had the same conclusion! 18 In the fundamental-adjoint plane of SU(2) lattice gauge theory with couplings $\beta_{F}$ and $\beta_{A}$, there is a well-known first-order phase transition which starts at the 4-d Z2 LGT transition (at $\beta_{A}=\infty$ ) and ends in the middle of the diagram at $\left(\beta_{F}, \beta_{A}\right)=(1.48,0.90) ! 19$ This has been seen as a critical point, below which an analytic path exists between the strong-coupling confining region with the weak-coupling continuum limit. However, that would require the first-order phase transition to be a non-symmetry-breaking transition. If it were symmetry-breaking, on the other hand, then the end of the first-order line would be a tricritical point, and the transition would have to continue as a higher-order one, bisecting the entire coupling plane into symmetry-broken and unbroken sectors, as above. Interestingly, a critical point and a tricritical point are rather easily distinguished through the scaling behavior of the attached first-order transition. In particular, the scaling relationship between the latent heat and the size of the metastability region is linear in the critical case and quadratic for the tricritical case ${ }^{18}$ One simply monitors the shape of the growing hysteresis rectangle in the energy-coupling plane while moving 
up the first-order line. The numerical evidence points convincingly to the tricritical case. 18 This again implies a symmetry-breaking phase transition separates the strong and weak-coupling regions. One of the strengths of this energy-scaling argument is that it does not require identification of the symmetry or order parameter involved, and no gauge-fixing is required. The tricritical case simply requires some symmetry to break. However, it seems likely it is the same symmetry breaking as studied above, something relatively easy to check.

\section{Artifact Driven Transition}

It is interesting to consider possible mechanisms which could drive the $\mathrm{SU}(2)$ transition. In the $\mathrm{U}(1)$ case, confinement arises from abelian monopoles, which are strongcoupling lattice artifacts. 20 The confined phase occurs when the monopoles form percolating loops. Confinement can be prevented by suppressing monopoles with a chemical potential. 21 Some time ago a gauge invariant monopole was introduced which could play the same role for the $\mathrm{SU}(2)$ theory ${ }^{7}$ It carries $\mathrm{SO}(3)$ and $\mathrm{Z} 2$ degrees of freedom which in some sense cancel each other, so was named an $\mathrm{SO}(3)-\mathrm{Z} 2$ monopole. Suppressing these monopoles, together with a positive plaquette restriction, appears to prevent the transition to the confining phase ${ }^{6}$ The system stays in the spontaneously magnetized phase all the way to zero coupling when this constraint is imposed ${ }^{22}$ Lattices to $60^{4}$ have been measured and the lattice spacing (determined from the running coupling) is such that these should definitely be in the confining region if universality applies. Potentials, measured to $1.5 \mathrm{fm}$, show no evidence of a linear term.

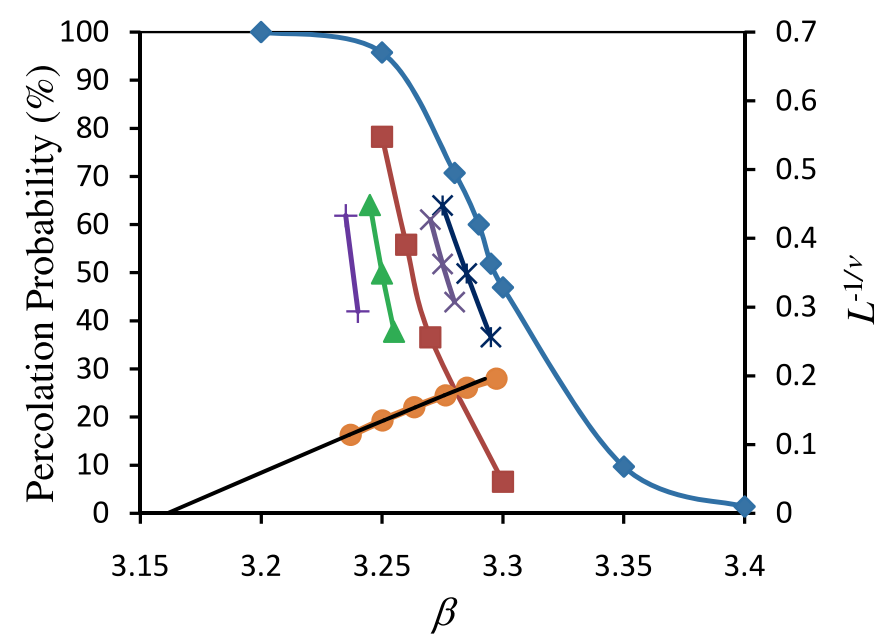

Fig. 3. Percolation probabilities for $\mathrm{SO}(3)-\mathrm{Z} 2$ monopoles as a function of $\beta$ for various lattice sizes (diamond $16^{4}, * 18^{4}, \times 20^{4}$, box $24^{4}$, triangle $30^{4},+40^{4}$ ). Also shown is extrapolation of $50 \%$ point to the infinite lattice (uses right $\mathrm{y}$-axis). 
It is interesting to monitor these monopoles in the standard Wilson theory on periodic lattices. They form percolating clusters in the crossover region where confinement occurs and de-percolate at weaker couplings. Fig. 3 shows the percentages of percolating lattices as a function of $\beta$ for various lattice sizes. The finite-lattice percolation transition can be taken to occur at the $50 \%$ level. Also shown is the extrapolation of the percolation transition to the infinite lattice. The extrapolation shown uses the finite-lattice shift equation for a higher-order phase transition 23

$$
1 / \beta_{c L}=1 / \beta_{c}+c L^{-1 / \nu}
$$

using the expected value $\nu=1.7$ from Ref. 6 . Here $L$ is the linear lattice size and $\beta_{c L}$ is the apparent critical point on the finite lattice. A fit to the data for $c$ and $\beta_{c}$ gives $\beta_{c}=3.161(2)$. If instead, $\nu$ is assumed to be unknown and determined from the fit then the best fit is obtained for $\nu=1.1$ yielding $\beta_{c}=3.193(1)$. Setting $\nu=0.5$ gives $\beta_{c}=3.226(2)$. The uncertainties in $\beta_{c}$ from the fixed- $\nu$ fits are much smaller than the differences in fits with different $\nu$ 's, so the majority of uncertainty in $\beta_{c}$ is from the extrapolation. Allowing a broad range for this exponent from 0.5 to 1.7 gives for the infinite lattice $\beta_{c}=3.19(3)$. It is very interesting that this is consistent with the position of the deconfining Coulomb-gauge magnetization transition determined from data-collapse fits to scaling behavior, which yielded an infinite lattice critical point of 3.18(8) ${ }^{6}$ It seems unlikely that these agree by mere coincidence. The percolation study was done after the other study was completed and released. Being that the percolation study was performed on the standard Wilson theory using conventional heat-bath and over-relaxation updates and with periodic boundary conditions, the coincidence of these results for $\beta_{c}$ gives additional confidence that the open boundary-condition Coulomb-gauge methods used in Ref. 6 are reliable. The fact that the percolation transition moves to smaller $\beta$ as the lattice size is increased means it almost certainly exists on the infinite lattice. The connection between $\mathrm{SO}(3)-\mathrm{Z} 2$ monopole percolation and confinement will be further explored in a forthcoming publication 22 .

\section{Conclusion}

It has long been known that lattice gauge theories have a lot in common with spin models. This becomes especially apparent in certain fixed gauges for which the remnant symmetry can be matched onto a spin model. The result presented above implies spin and gauge theories have even more in common, namely long range ferromagnetic order. This has important consequences for the non-abelian lattice gauge theories $\mathrm{SU}(2)$ and $\mathrm{SU}(3)$ which were previously assumed to be exceptions to this behavior. The relatively large critical exponent, $\nu \sim 1.7$ for $\mathrm{SU}(2)$, explains how such a transition could have been missed, since the corresponding specific heat singularity is very soft.

The ordered continuum limit means that the quenched lattice gauge theory without fermions does not confine in the continuum limit, an idea which has been 
suggested previously ${ }^{24 \mid 25]}$ Because good phenomenological evidence exists for quark confinement, however, some source of confinement must be sought, which need not necessarily be a linear potential. If light quarks are added to the theory, chiral symmetry is still expected to break spontaneously through the formation of a quark condensate. This only requires a sufficiently strong force, not necessarily a confining one. If this collective state polarizes in such a way as to repel strong color fields, the chiral condensate could form a kind of bag surrounding mesons and baryons, contributing a confining-like term to the force over a limited range, though confinement would not be absolute. ${ }^{26|27| 28}$ Also, if a quark were to find itself a long distance from its partner antiquark, a quark/antiquark current in the chiral condensate could quickly generate local partners. This is somewhat different from generating quark anti-quark pairs from gluon "sparking" which originate at the same location, but that too can prevent isolated quarks from existing provided there is sufficient energy in the bond. Thermodynamics of the quark-gluon plasma is also modified in this picture, since confinement is no longer in the gluon sector. A phase transition or at least a rapid crossover to a chiral-symmetric phase at high physical temperatures will undoubtedly still exist, which, if chiral symmetry breaking is related to confinement as above, will also be deconfining.

\section{References}

1. J. Kogut, Rev. Mod. Phys. 51, 659-713 (1979).

2. F.J. Wegner, J. Math. Phys. 12, 2259-2272 (1971).

3. D. Zwanziger, Phys. Rev. Lett. 90, 102001 (2003).

4. J. Greensite, S. Olejník, and D. Zwanziger, Phys. Rev. D 69, 074506 (2004).

5. E. Marinari, M. Paciello, G. Parisi, and B. Taglienti, Phys. Lett. B 298, 400-404 (1993).

6. M. Grady, arXiv:1104.3331 [hep-lat].

7. M. Grady, hep-lat/9806024 M. Grady, Nucl. Phys. B (Proc. Suppl.) 73, 551-553 (1999).

8. E.T. Tomboulis, Phys. Rev. Lett. 50, 885-888 (1983).

9. E.T. Tomboulis, arXiv:0707.2179 [hep-th].

10. K.R. Ito and E. Seiler arXiv:0711.4930 [hep-th].

11. B. Durhuus and J. Fröhlich, Commun. Math. Phys. 75, 103-151 (1980).

12. J. Fröhlich, B. Simon, and T. Spencer, Commun. Math Phys. 50, 79-85 (1976).

13. K. Kanaya and S. Kaya, Phys. Rev. D 51, 2404-2410 (1995).

14. A.D. Beath and D.H. Ryan, J. Appl. Phys. 97, 10 A506 (2005).

15. M. Grady, arXiv:0907.4102 [hep-lat].

16. P.M. Chaiken and T.C. Lubensky, Principles of Condensed Matter Physics (Cambridge University Press 1995), p173.

17. W. Rudin, Principles of Mathematical Analysis, second ed. (McGraw Hill, 1964), p89.

18. M. Grady, Nucl. Phys. B 713, 204-218 (2005).

19. G. Bhanot and M. Creutz, Phys. Rev. D 24, 3212-3217 (1981).

20. T.A. Degrand and D. Toussaint, Phys. Rev. D 22, 2478-2489 (1980).

21. V.G. Bornyakov, V.K. Mitrjushkin, and M. Muller-Preussker, Nucl. Phys. B (Proc. Suppl.) 30, 587-590 (1995).

22. M. Grady, to appear. 
23. M.N. Barber, in Phase Transitions and Critical Phenomena, eds. C. Domb and J.L. Lebowitz (Academic Press, 1983), Vol. 8, pp. 146-266.

24. M. Grady, Z. Phys C 39, 125-136 (1988).

25. A. Patrascioiu, E. Seiler, and I.O. Stamatescu, Nuovo Cimento D 11, 1165-1190 (1989); A. Patrascioiu, E. Seiler, V. Linke, and I.O. Stamatescu, Nuovo Cim. 104B, 229-236 (1989).

26. M. Grady, Nuovo Cim. 105A, 1065-1079 (1992).

27. K. Cahill and G. Herling, Nucl. Phys. B Proc. Suppl. 73, 886-888 (1999); K. Cahill, hep-ph/9901285

28. A somewhat different scenario in which quarks are responsible for confinement is given in V.N. Gribov, Phys. Scr. T15, 164-168 (1987), Phys. Lett. B 194, 119-124 (1987); J. Nyiri, (Ed.), The Gribov Theory of Quark Confinement (World Scientific, 2001). 